\title{
Understanding the Allocation of Caesarean Outcome to Provider Type: A Chart Review
}

\section{Comprendre la répartition des résultats de césariennes en fonction du type de dispensateur de services : examen des dossiers}

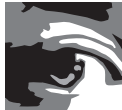 \\ KELLIE THIESSEN, RM, RN, PHD \\ Director, Midwifery Program (University of Manitoba/McMaster University) \\ Assistant Professor, College of Nursing, Rady Faculty of Health Sciences \\ University of Manitoba \\ Winnipeg, $M B$ \\ NATHAN NICKEL, MPH, PHD \\ Assistant Professor, Manitoba Centre for Health Policy \\ Max Rady College of Medicine, Rady Faculty of Health Sciences \\ University of Manitoba \\ Winnipeg, $M B$ \\ HEATHER J. PRIOR, MSC \\ Manitoba Centre for Health Policy \\ Max Rady College of Medicine, Rady Faculty of Health Sciences \\ University of Manitoba \\ Winnipeg, $M B$ \\ MARGARET MORRIS, MD, FRCSC, MED \\ Professor, Department of Obstetrics, Gynecology and Reproductive Science \\ University of Manitoba \\ Health Sciences Centre, Women's Hospital \\ Winnipeg, $M B$ \\ KRISTINE ROBINSON, BSCN, RM, MSC \\ Winnipeg Regional Health Authority \\ Winnipeg, $M B$
}




\begin{abstract}
Introduction: The concept, "most responsible provider" has a specific definition in the Canadian National Discharge Abstract Database (DAD). Variation exists in how care providers are defined in administrative data.

Methods: We compared chart data with administrative data to understand how "most responsible provider" was identified in these two data sources.

Results: We found a 3\% discrepancy between data sources. Differences between data sources were attributable to transfers in care that occurred at birth.

Discussion: "Most responsible provider" should consider the full trajectory of care when assigning outcomes in order to understand how to best support optimal health among lowrisk births.
\end{abstract}

\title{
Résumé
}

Introduction : La définition du concept de « dispensateur principal » est précisée dans la Base de données sur les congés des patients (BDCP). Or, il existe certaines variations quant à la façon de définir les dispensateurs de services de santé dans les données administratives. Méthode : Nous avons comparé les données des dossiers aux données administratives pour comprendre comment le dispensateur est désigné dans ces deux sources de données.

Résultats : Nous avons observé une divergence de $3 \%$ entre les sources de données. Ces différences sont imputables aux transferts des soins qui ont lieux lors des naissances.

Discussion: La notion de « dispensateur principal » doit tenir compte de l'ensemble de la trajectoire des soins au moment d'assigner les résultats, et ce, afin de comprendre la façon d'optimiser la santé dans les cas de naissances à faible risque.

\section{Introduction}

The concept of "most responsible provider" (MRP) is a data description, defined in the Canadian National Discharge Abstract Database (DAD) as: "The most responsible provider ... a physician or other provider (i.e., midwife) who is responsible for the care/treatment of the patient for the majority of visits in healthcare facilities (p. 192)" (CIHI 2017). MRP is assigned based on the point of care in these acute care facilities, which are typically hospitals. The data element of MRP in the DAD is standard for reporting, however, researchers have defined MRP in studies differently (Aubrey-Bassler et al. 2015).

The majority of maternity outcome studies use administrative data to answer research questions (Birthplace in England Collaborative Group 2011; Heaman et al. 2012; Hutton et al. 2009; Thiessen et al. 2016). While administrative data are reliable and inform trends in population health (Harvey et al. 1996; Janssen et al. 2007, 2009), there are limitations such as incomplete data or coding discrepancies/disagreements (MCHP 2017). Additionally, based on availability of the data, it may not be possible to fully understand all processes 
preceding interventions such as availability of technology, level of provider's skill or style, out of hospital birth, and philosophy of practice (Baruffi et al. 1990; Janssen et al. 2007).

Most perinatal outcomes studies only consider outcomes around the time of birth (Birthplace in England Collaborative Group 2011; Harvey et al. 1996; Janssen et al. 2007; Janssen et al. 2009; Sword et al. 2015; Thiessen et al. 2016). In order to understand differences in models of care, we believe prenatal care should be considered. Prenatal care has been shown to impact outcomes, and the quality of prenatal care has been shown to be a significant factor in perinatal health (Sword et al. 2012, 2015).

The results from our pilot study (Thiessen et al. 2016) whereby the DAD definition of MRP was used to allocate MRP to outcomes, demonstrated that midwives had a 1.7\% Caesarean section rate, which was low compared to rates in literature (Birthplace in England Collaborative Group 2011; Harvey et al. 1996; Janssen et al. 2007, 2009). We wanted to investigate any potential discrepancy between chart data and administrative data. Therefore, the objective of this study was to understand if the primary outcome of Caesarean section, in our pilot study, was misallocated to the MRP. Our two research questions were: 1) How does the assignment of MRP differ between chart data and administrative data reports; 2 ) What percentage of Caesarean sections were incorrectly allocated?

\section{Methods}

This is a descriptive study. We conducted a retrospective chart review to understand how provider type had been assigned the outcome of Caesarean section. We randomly selected Caesarean section cases from the cohort in the administrative data from our pilot study (Thiessen et al. 2016) and matched them to their prospective charts in each facility to understand how provider type was allocated to the Caesarean section outcome. In cases where the administrative data disagreed with the chart data, we were interested in understanding variables around each case that might have influenced how the outcomes were allocated.

\section{Inclusion Criteria}

\section{COHORT DEFINED}

We used these defined low-risk (College of Midwives of Manitoba 2011; Health Sciences Centre 2014; Janssen et al. 2009; St. Boniface General Hospital n.d.) cases from our administrative data and linked them to their charts.

\section{MATERNITY PROVIDER TYPES IN MANITOBA}

In Manitoba, midwives and general/family practice physicians consult with and transfer to obstetricians when indicated. Obstetricians do the majority of maternity care and exepct for rural areas (general/family practice and general surgeons responsible for Caesarean sections), are responsible for the majority of Caesarean sections. Midwives are responsible for low-risk women and do not perform surgical procedures. 


\section{Primary Outcome of Interest}

Our primary outcome of interest was the allocation of provider type to the Caesarean section outcome by the two major tertiary care centres responsible for all the hospital deliveries in the Winnipeg Regional Health Authority (WRHA) of Manitoba. We reviewed charts from 2004/05 to 2012/13. During this time period, there were a total of 132,918 births in Manitoba. Of those births, 47,083 were identified as high risk (35\%) and 85,835 (65\%) were identified as low risk. The total number of Caesarean sections from the low-risk birth cohort during this time frame for the two urban tertiary care centres was: Facility $1(n=3,563)$ and Facility $2(\mathrm{n}=4,158)$.

\section{Data Collection}

\section{ADMINISTRATIVE DATA AND DEFINITION OF VARIABLES}

Caesarean section data came from the low-risk cohort of women from our previous pilot study (Thiessen et al. 2016). The data came from three databases housed in the Population Research Data Repository (MCHP 2017). The hospital abstract database in the Manitoba Population Research Data Repository at MCHP codes provider type as follows: 1) Specialty of provider who actually delivers the baby;2) Specialty of attending provider; and 3) Specialty of MRP, i.e., the physician responsible for the primary (most responsible) patient service that reflects the definition for MRP as delineated in the national DAD. Therefore, in the administrative data cases, the provider most responsible for the primary (most responsible) patient service at birth was attributed to the outcome of interest.

\section{CHART REVIEW DATA AND DEFINITION OF VARIABLES}

To gain an understanding of how the outcome of Caesarean section was attributed to the MRP type in the chart data, we abstracted: provider type (before birth, at birth, at discharge, and for prenatal care), mode of delivery (Caesarean section), gestation at first prenatal visit and at transfer of care, transfer of care, and reason for transfer of care.

The chart reviews took place at two urban tertiary care centres in Winnipeg, Manitoba. During our study time frame, there were only 35 Caesarean sections allocated to midwives between both facilities. We included all midwifery Caesarean cases. We formed a matched-comparison group using a 3:1 (family practice) and 5:1 (OB/GYN) matching ratio. Comparisons were matched by month of birth and birth facility. A chart audit tool was devised and piloted by the research team prior to commencing the full chart review study.

A discrepant case in our study was then defined as a case where the provider type was allocated to a Caesarean section in the chart data, but that same case differed with how provider type was allocated to Caesarean in the administrative database. In summary, discrepant cases occurred when the chart disagreed with the administrative database. 


\section{Analysis}

After linking the chart review data with the administrative data cases at the MCHP, we performed descriptive statistics to identify what proportion of these cases differed between data sources in how the MRP type was allocated to the outcome of Caesarean section. We used the kappa statistic to measure the agreement for categorical (grouped) data. The kappa looks for agreement across the diagonal of the table and will return a high score if there is high agreement between the two variables (McHugh 2012). We identified the proportion of cases for each provider type - midwife, family physician, and obstetrician. We calculated the proportion of discrepant cases for each provider-type at each point during the birth process. Chi-square tests were done to analyze the agreement by provider type (Y/N) and Facility (1 and 2). All analyses used an a priori statistical significance level of $p<0.05$ and were conducted using SAS 9.4.

\section{Ethics}

All research assistants involved in the chart review completed the orientation and signed the Personal Health Information Act pledge. Ethics approval was obtained from the University of Manitoba Health Research Ethics Board (HS 19004 [H2015:382]). A research agreement was obtained to access Manitoba information at the MCHP. Approvals were obtained from both the Impact Committee from tertiary care centre number one (RI2015:154) and from the Review Committee of tertiary care centre number two (RRC/2015/1519). Finally, approval was obtained from the Manitoba Health Information Privacy Committee (2015/2016-54).

\section{Results}

We reviewed a total of 315 charts from tertiary care centre number one $(n=153)$ and tertiary care centre number two $(n=162)$ (See Table 1$)$.

\section{Inter-Rater Reliability}

To ensure consistency among chart reviewers, we randomly selected $10 \%$ of the charts in our study sample to examine the reproducibility of extracting the data elements by independent reviewers. The review was independently completed by the two trained research assistants who also completed the overall chart review. Between the two trained research assistant reviewers, we measured the inter-rater reliability (IRR) with all data elements included on the chart audit tool using the percent level of agreement (McHugh 2012). The IRR, as measured by percent agreement, was initially $77.4 \%$ for mode of delivery and $67.7 \%$ for healthcare provider type at birth. We learned that some family practice physicians have obstetrical training and are coded as obstetricians. We were able to identify all these cases. After review and revision of the chart audit methods and tool, the data that were extracted from all charts included in the study and the percent level of agreement on all variables was $100 \%$.

Table 1 describes the percentage of agreement between the MRP type in the chart data and the administrative data. For example, when an OB/GYN was identified as the MRP for 
Understanding the Allocation of Caesarean Outcome to Provider Type: A Chart Review

the provider at birth in the administrative data, there was $100 \%$ agreement with the chart review data.

TABLE 1. Low-risk cohort Caesarean sections by facility: data collection facility I \& 2 .

Time period: 2004/05 to 2012/13

\begin{tabular}{|l|l|l|l|}
\hline \multicolumn{2}{|l|}{$\begin{array}{l}\text { Total Caesarean } \\
\text { sections (n) }\end{array}$} & Matched & $\begin{array}{l}\text { Total \# charts } \\
\text { for review }\end{array}$ \\
\hline Tertiary care centre I & 3,461 & $5: 1$ & 85 \\
\hline OB/GYN & 85 & $3: 1$ & 51 \\
\hline FP & 17 & & \\
\hline MW & \multicolumn{3}{|l|}{} \\
\hline Total [tertiary care centre I] & 3,897 & $5: 1$ & 90 \\
\hline Tertiary care centre 2 & 243 & $3: 1$ & 54 \\
\hline OB/GYN & 18 & & \\
\hline FP & & & $\mathbf{1 6 2}$ \\
\hline MW & & $\mathbf{3 1 5}$ \\
\hline Total [tertiary care centre 2] & & & \\
\hline TOTAL \# charts for review & & & \\
\hline
\end{tabular}

TABLE 2. Agreement of provider assignment between administrative data and chart review data (combined facilities)

\begin{tabular}{|c|c|c|c|}
\hline MRP administrative data & Chart review data & Percent agreement (\%) & Kappa $(95 \%$ Cl) \\
\hline OB/GYN & Provider at birth & 100 & \multirow{3}{*}{$0.026(-0.003,0.054)$} \\
\hline FP & Provider at birth & $s^{*}$ & \\
\hline MW & Provider at birth & $0^{* *}$ & \\
\hline OB/GYN & Provider at discharge & 98.27 & \multirow{3}{*}{$0.947(0.912,0.981)$} \\
\hline FP & Provider at discharge & 94.68 & \\
\hline MW & Provider at discharge & 97.14 & \\
\hline OB/GYN & Provider at birth & 99.43 & \multirow{3}{*}{$0.965(0.937,0.993)$} \\
\hline FP & Provider at birth & 96.91 & \\
\hline MW & Provider at birth & 94.12 & \\
\hline OB/GYN & Provider of prenatal care & 92.68 & \multirow{3}{*}{$0.904(0.858,0.950)$} \\
\hline FP & Provider of prenatal care & 97.80 & \\
\hline MW & Provider of prenatal care & 94.29 & \\
\hline
\end{tabular}

\footnotetext{
*"s" denotes suppressed values for confidentiality reasons. Note that missing chart review data were not included in percent agreement calculations; percentages may not reflect total sample size.

***Midwives do not perform Caesarean sections, therefore, this value was 0
} 
In 11 cases, we noted misalignment between the chart review data and the administrative data. In these cases, for example, at the time of birth, the chart data showed a different MRP than the administrative data. It was interesting to note, however, that for each of these cases $(n=11)$ where this misalignment occurred, the provider prior to birth had at least 5 or more prenatal care visits. Reasons for transfer are summarized in Table 2.

\section{Discussion}

Our findings revealed a total of 11 cases where obstetricians were assigned a Caesarean section in the administrative data when, in fact, the chart data revealed they had not been responsible for the prenatal care for that client. Among those 11 cases, 10 were transfers of care that continued to code OB/GYN as MRP after transfer, in spite of the fact that the majority of the overall care was done by another provider.

In the misallocated cases, we discovered greater than $2 / 3$ of the prenatal visits ( $>5$ prenatal visits) were done by the provider at birth. Provider type for the majority of prenatal care has implications beyond the DAD definition of MRP (CIHI 2017) and evidence supports this (Heaman et al. 2012). Prenatal care is considered a preventive health service that impacts the health outcomes of women and infants (Heaman et al. 2014). The MRP type definition should consider the provider responsible for the majority of prenatal care, as well.

It is also noteworthy that elective Caesarean section cases were allocated to OB/GYN when in fact they had been cared for by either a midwife or family physician prenatally.

While we matched a small random sample from our larger cohort study, these findings have implications for clinical practice, including an increased understanding of how we might provide more clarity around roles of providers. It is important to consider which provider had the majority of the prenatal care when assigning MRP since this will influence the study results.

Our results provide a heightened awareness regarding transfer of care and the misallocation of provider type, and of the need for more clarity and consistency in this process.

\section{Limitations}

Factors that lead to misallocation of provider type in the administrative data may also be related to birth outcomes. However, given the low rate of misalignment, this is likely a minor concern.

Limitations of our study include time and financial constraints. Due to time and financial constraints, we could not fully execute a validation study. Additionally, the DAD does not include out of hospital birth data, which limits further understanding of full scope midwifery practice. Another limitation is that there could have been data entry error during the abstraction and/or re-abstraction process; however, data abstractors at the two institutions go through a 2-year training and the administrative data have been extensively validated in 
numerous studies. We also used two re-abstractors with high inter-rater agreement between them; thus, we think this limitation poses minimal threats to our study.

\section{Summary}

There was approximately a 3\% discrepancy in allocating a Caesarean section to obstetricians in our sample. It is important to consider which provider had the majority of the prenatal care. As policy makers consider health workforce planning, understanding who is providing care at what point is important in planning care teams for health delivery services. Therefore, understanding the details of how providers are allocated to outcomes in population health studies is essential in understanding the roles each profession has in meeting the needs of population health. Otherwise, data discrepancy misleads decision-makers about the contribution of each healthcare professional involved in care delivery.

Examples exist of validation studies that use the DAD to understand coding with hospital databases (Heaman et al. 2012; McHugh 2012) and linkage of census data to the DAD (Heaman et al. 2014). To date, there are no validation studies that analyze MRP assignment based on the DAD and hospital charts. Our next step involves using a large sample size to triangulate three data sources (MCHP 2017) to determine a "gold standard dataset" to maximize the probability of any one outcome occurring by the MRP and to evaluate the completeness of the data in each source (Sword et al 2012). Assigning the MRP to outcomes is potentially misleading if limitations aren't highlighted regarding the definition and its usage in any given study.

\section{Acknowledgements}

The authors acknowledge the Manitoba Centre for Health Policy for the use of data contained in the Population Health Research Data Repository under project \#2016-002 (HIPC\#2015/2016-54). The results and conclusions are those of the authors and no official endorsement by the Manitoba Centre for Health Policy, Manitoba Health, Healthy Living, and Seniors, or other data providers is intended or should be inferred. This study was funded by the University Start-up Funds for New Faculty at the University of Manitoba.

Correspondence may be directed to: Dr. Kellie Thiessen, College of Nursing, Rady Faculty of Health Sciences, University of Manitoba, 89 Curry Place, Winnipeg, MB R3T 2N2; tel.: 204474-6684; e-mail: Kellie.thiessen@umanitoba.ca.

\section{References}

Aubrey-Bassler, K., R. Cullen, A. Simms, S. Asghari, J. Crane, P. Wang and M. Godwin. 2015. “Outcomes of Deliveries by Family Physicians or Obstetricians: A Population-Based Cohort Study Using an Instrumental Variable." Canadian Medical Association Journal 187(15): 1125-32. doi: http://doi.org/10.1503/cmaj.14163.3 Baruffi, G., D.M. Strobino and L.L. Paine. 1990. "Investigation of Institutional Differences in Primary Caesarean Birth Rates." Journal of Nurse-Midwifery 35(5): 274-81. 
Birthplace in England Collaborative Group. 2011. "Perinatal and Maternal Outcomes by Planned Place of Birth for Health Women with Low Risk Pregnancies: The Birthplace in England National Prospective Cohort Study." BMJ 343: 1-13. doi10.1136/bmj.d740.0

Canadian Institute for Health Information (CIHI). 2017. “DAD Abstracting Manual.” Retrieved February 22, 2017. <https://secure.cihi.ca/estore/productSeries.htm?pc=PCC7>.

College of Midwives of Manitoba (CMM). 2011. Standard for Out of Hospital (OOH) Birth. Retrieved June 30, 2018. <http://midwives.mb.ca/policies_and_standards/8\%20Standard\%20for\%20Planned\%20Out $\% 20$ of $\% 20$ Hospital\%20Birth.pdf>.

Harvey, S., J. Jarrell, R. Brant, C. Stainton and D. Rach. 1996. “A Randomized, Controlled Trial of NurseMidwifery Care." Birth 23(3): 128-35.

Health Sciences Centre. 2014. "Practice Guidelines Women's Health Program." Obstetrics: Perinatal Bed Utilization. Section 80.275.010.

Heaman, M., D. Kingston, M.E. Helewa, M. Brownell, S. Derksen, B. Bogdanovic et al. 2012. “Perinatal Services and Outcomes in Manitoba." Retrieved February 20, 2017. <http://mchp-appserv.cpe.umanitoba.ca/ reference/perinatal_report_WEB.pdf>.

Heaman, M., M. Moffatt, L. Elliot, W. Sword, M.E. Helewa, H. Morris et al. 2014. “Barriers, Motivators and Facilitators Related to Prenatal Care Utilization among Inner-City Women in Winnipeg, Canada: A CaseControl Study." BMC Pregnancy and Childbirth 14: 227. doi.org/10.1186/1471-2393-14-227.

Hutton, E.K., A.H. Reitsma and K. Kaufman. 2009. "Outcomes Associated with Planned Home and Planned Hospital Births in Low-Risk Women Attended by Midwives in Ontario, Canada, 2003-2006." Birth 35(3): 180-09. doi: 10.1111/j.1523-536X.2009.00322.x

Janssen, P.A., E.M. Ryan, D.J. Etches, M.C. Klein and B. Reime. 2007. “Outcomes of Planned Hospital Birth Attended by Midwives Compared with Physicians in British Columbia." Birth 34(2): 140-07.

Janssen, P.A., L. Saxell, L.A. Page, M.C. Klein, R.M. Liston and S.K. Lee. 2009. “Outcomes of Planned Home Birth with Registered Midwife versus Planned Hospital Birth with Midwife or Physician." Canadian Medical Association Journal 181(6-7): 377-83. doi: 10.1503/cmaj.081869.

Manitoba Centre for Health Policy (MCHP). 2017. "Population Health Research Data Repository." Retrieved June 6, 2018. <http://umanitoba.ca/faculties/health_sciences/medicine/units/chs/departmental_units/mchp/ resources/repository/datalist.html>.

McHugh, M.L. 2012. “Interrater Reliability: The Kappa Statistic.” Biochem Med (Zagreb) 22(3): 276-82.

St. Boniface General Hospital, LDRP Unit. (n.d.) Guideline for Admission to the SBGH LDRP Unit.

Sword, W., M. Heaman, M.A. Biro, C. Homer, J. Yelland, N. Akhtar-Danesh and A. Bradford-Janke. 2015.

"Quality of Prenatal Care Questionnaire: Psychometric Testing in an Australia Population." BMC Pregnancy and Childbirth 15: 214. doi: 10.1186/s12884-015-0644-7.

Sword, W., M. Heaman, S. Brooks, S. Tough, P. Janssen, D. Young et al. 2012. “Women's and Care Providers' Perspectives of Quality Prenatal Care: A Qualitative Descriptive Study." BMC Pregnancy and Childbirth 12: 29. doi: https://doi.org/10.1186/1471-2393-12-2.9.

Thiessen, K., N. Nickel, H. Prior, A. Banerjee, M. Morris and K. Robinson. 2016. "Maternity Outcomes in Manitoba Women: A Comparison between Midwifery-Led Care \& Physician-Led Care at Birth." Birth 43(2): $108-15$. 